\title{
An Enhanced Recursive Median Filter for Noise Reduction based on Randomness in Pixel Values of an Image
}

\author{
Priya Varshney \\ Department of Computer Science and Engg., \\ PSIT, Kanpur
}

\author{
Anshuman Tyagi \\ Associate Professor, \\ Department of computer Science and Engg., \\ PSIT, Kanpur
}

\begin{abstract}
Noise is a prominent factor which when added in an image reduces its quality. So to enhance the quality of an image, removing noise is required which is an important field of research. Many researchers have proposed various algorithms and compared their result. This paper focuses on removing noise from distorted image using Recursive Median Filter. Recursive Median Filter (RMF) is one of the noise reduction technique used for removing impulse noise. To enhance the performance of RMF using the concept of degree of randomness i.e. Entropy, a novel approach is introduced named as Enhanced Recursive Median Filter (ERMF). In the proposed filter, the size of moving window is changed based on the entropy value of moving window. Result shows that the proposed filter enhance the performance in removing the noise as compared to recursive median filter.
\end{abstract}

\section{Keywords}

Digital Image Processing, Median Filter, Recursive Median Filter, Randomness.

\section{INTRODUCTION}

Image Noise is random (not present in the object imaged) variation of brightness or color information in images and is usually an aspect of electronic noise. The principal sources of noise in the digital image are:

a) The imaging sensor may be affected by environmental conditions during image acquisition.

b) Insufficient Light levels and sensor temperature may introduce the noise in the image.

c) Interference in the transmission channel may also corrupt the image.

d) If dust particles are present on the scanner screen, they can also introduce noise in the image [1].

Noise removal is one of the major parts in the field of image processing. Impulse noise removal can be an extreme goal to improve the quality of images. The goal of removing impulsive noise is primarily to suppress the noise as well as to preserve the integrity of edges and detailed information like structural features, textural information. Impulse noise corruption is very common in digital images. Various Filters are used to denoising digital image and the important thing is to preserve details of image. A very common nonlinear filter which is used for smoothing images is Median Filter [2]. A major advantage of the median filter over linear filters is that the median filter can eliminate the effect of input noise values with extremely large magnitudes. Median Filter reduces noise from an image without blurring and edge destruction [2-3]. Median Filter is an efficient filter for removing noising as it has low computation. To improve the performance of Median Filter, various enhancements of Median Filter are proposed, i.e. Adaptive Median Filter [7], Weighted Median Filter [6], Adaptive Weighted Media Filter [5], Recursive Median Filter $[4,9]$ etc. Median Filter is implemented to the corrupted image using a window which is moved across the input image and the central pixel of the window is replaced by the output of median filter which is the median value of the pixels within the window [3]. One of the most efficient modifications of median filter is Recursive Median Filter (RMF) [4, 9]. In the Recursive Median Filter, similar to median filter, the center pixel of the window is replaced by the median value of all the pixels inside the window but the obtained values (i.e., previous outputs of RMF) for some pixels of window are used instead of the pixel values. The recursive property of Recursive Median Filter causes that the Recursive Median Filter has efficient noise removing capability than median filter [9]. The size of moving window has a significant effect on the performance of recursive median filter in reduction of noise in image. When an image has high density of noise and small size window is used for filtering then probability of selecting noisy pixel increases and performance of filter degraded. Moreover, using a large size of moving window can lose the details of image and provide a vague image. Therefore, choosing the optimal size of moving window is important and can increase the performance of recursive median filter. In this paper, by using the concept of randomness (also entropy) in value of pixels inside the moving window, the size of moving window is changed adaptively. Hence, when the entropy value is high of the moving window then select a large size of moving window for the recursive median filter as, in this situation, the number of pixels in the moving window increases so the probability of selection a noisy pixel as the output of recursive median filter decreases. On the other side, same size of moving window is used when the entropy value is low. Rest part of paper is organized as follows. Section 2 has concise description of Median filter and Recursive Median Filter. In Section 3 proposed filter is presented. In Section 4 results are shown. Conclusion is presented in section 5 .

\section{MEDIAN FILTER AND RECURSIVE MEDIAN FILTER}

The median filter is a non linear digital filter which is often used in digital image processing to reduce noise in an image. For small to moderate levels of salt and pepper noise the median filter has shown to be useful in reducing noise while preserving edges. The pattern of neighbors is called the "window" or "mask", which slides, pixel by pixel over the entire input image. The centre pixel is replaced by the median of the pixel values of the window. In one dimensional median filter, if $\mathrm{X}_{\mathrm{i}}$ is the input, the output $\mathrm{Y}_{\mathrm{i}}$ is given by: 
$Y_{i}=\operatorname{median}\left\{X_{i-n}, X_{i-n+1, \ldots \ldots . .,} X_{i+n}\right\}$

where the window have $2 \mathrm{n}+1$ pixels. The Recursive Median Filter is an enhanced median filter defined in eq 1 in which for filtering uses previous output of median filter rather than pixel values of input image. In one dimensional Recursive median filter, if $X_{\mathrm{i}}$ is the input, the output $\mathrm{Y}_{\mathrm{i}}$ is given by:

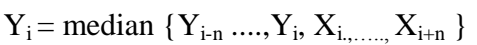

The Recursive median filter can extract image/signal roots better than median filtering in some applications such as image noise reduction [9].

\section{PROPOSED FILTER}

As per the description in the section 2,the performance of the recursive median filter depends on the size of the moving window. When an image has high density of noise and small size window is used for filtering then probability of selecting noisy pixel increases and performance of filter degraded. Moreover, using a large size of moving window can lose the details of image and provide a vague image. Therefore enhancing the performance of recursive median filter, the window size must not be same for whole image. The size of the moving window should be adjusted as per the noise density or randomness of image. Entropy is a statistical measure of randomness that can be used to characterize the texture of the input image [3] and is defined as:

$\mathrm{E}=-\sum_{k=1}^{K} \mathrm{P}\left(a_{k}\right) \log \mathrm{P}\left(a_{k}\right)$

Where $\left\{P\left(a_{1}\right), P\left(a_{2}\right), \ldots \ldots, P\left(a_{k}\right)\right\}$ is a set of probabilities which is associated to a source of random events $\left\{a_{1}, a_{2}, \ldots . ., a_{k}\right\}$. An image is elucidated as specimen of gray level source. The probabilities of source can be exhibited by the grey level histogram of a digital image. By using equation 3 the randomness in an image can be found. The moving window has pixel values and to find the randomness of these pixel value use the equation of entropy. Now in the proposed filter use the concept of uncertainty and adapt the size of the moving window of recursive median filter. As if uncertainty is high then using large moving window is beneficial as it decreases the selection of noisy pixel. On the other side if uncertainty is low then using small size moving window is profitable so as to increase the performance of recursive median filter. There should be a threshold value of uncertainty to decide the size of moving window. In proposed filter the threshold value is 0.6 . If the measure of uncertainty is less than or equal to 0.6 then the size of moving window is of $3 * 3$ and if the measure of uncertainty is greater than 0.6 then the size of moving window is $5 * 5$.

\section{RESULTS}

The performance of proposed filter is check on noisy images corrupted with salt and pepper noise. Two performance metrics on the basis of which comparisons are done are PSNR (Peak Signal to Noise Ratio) and MSE (Mean Square Error)

$\mathrm{PSNR}=10 \log _{10}\left(\mathrm{MAX}^{2} / \mathrm{MSE}\right)$

$\mathrm{MSE}=1 / \mathrm{MN} \sum_{i=1}^{M} \sum_{j=1}^{N}\left(x_{i j}-\bar{x}_{i j}\right)$

Where $\mathrm{M}$ and $\mathrm{N}$ are the dimension of image, $x_{i j}$ is the pixel value at position $\mathrm{i}$ and $\mathrm{j}$ of the original image and $\bar{x}_{i j}$ is the pixel value at position $i$ and $j$ of the filtered image.MAX is maximum possible pixel value of image. The original two images are shown in fig 1 . Each image is corrupted with salt and pepper noise of different density. Performance metrics (MSE and PSNR) of proposed filter is compare with the result of median filter, recursive median filter and are shown in fig 2 and 3. Filtered images using median filter, recursive median filter and proposed filter are also shown in fig 4 and 5 . The result shows that proposed filter performs better than median filter and recursive median filter.

\section{CONCLUSION}

In this paper a filter is proposed to reduce the salt and pepper noise in an image. In the proposed filter the randomness of pixel values in moving window is calculated and on the basis of that value the size of moving window is adapted. Therefore the performance of proposed filter increases as compared to median filter and recursive median filter with preserving details.

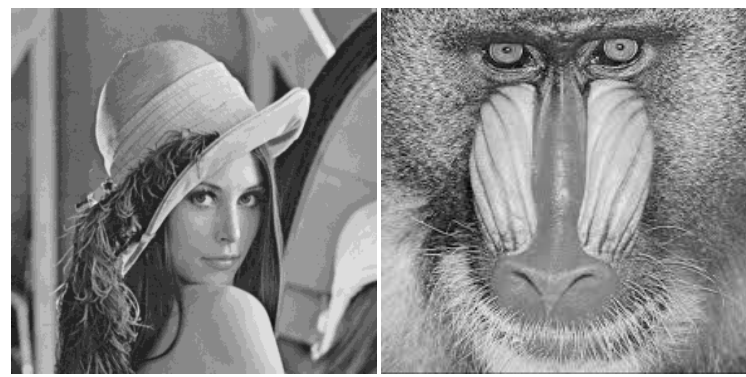

Fig 1: Original Images

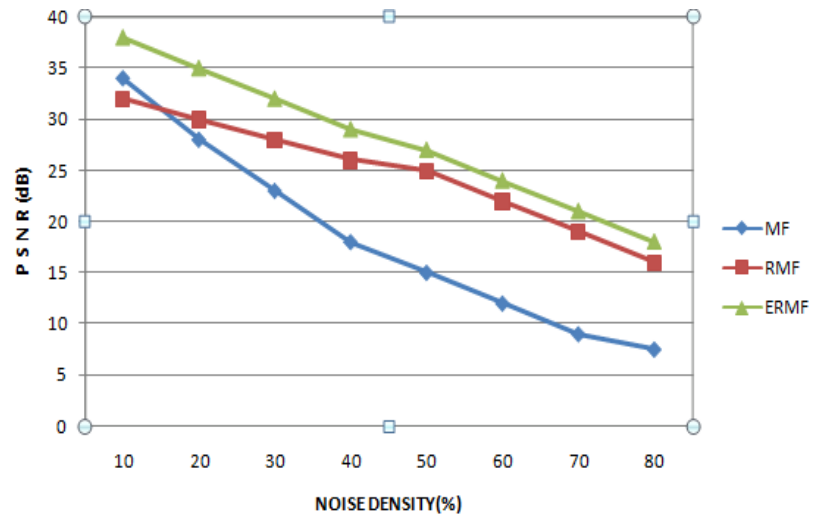

Fig 2: The Peak Signal to Noise Ratio of filters

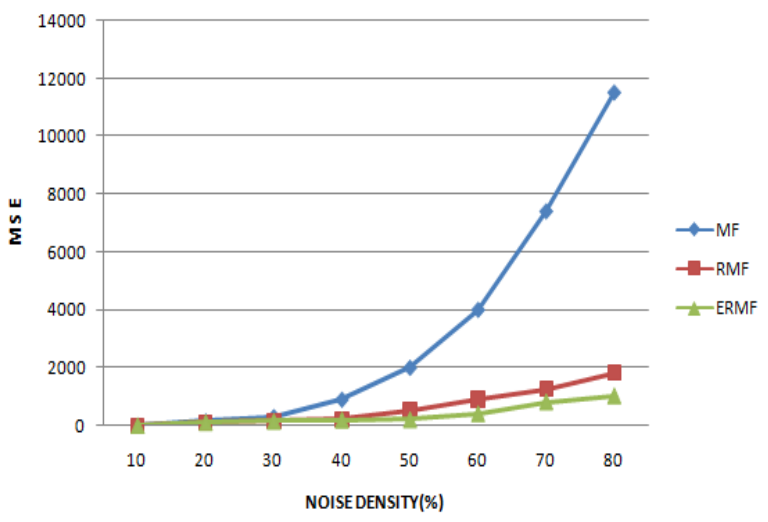

Fig 3: The Mean Square Error of filters 


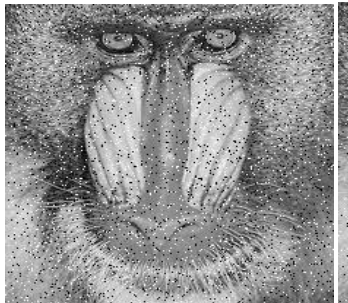

(a)

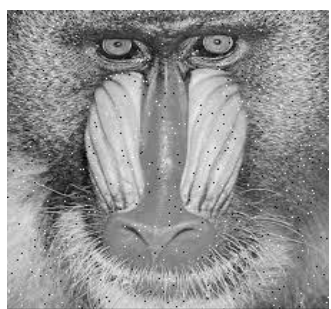

(c)

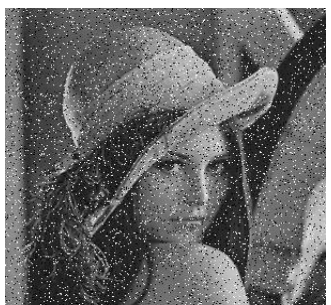

(a)

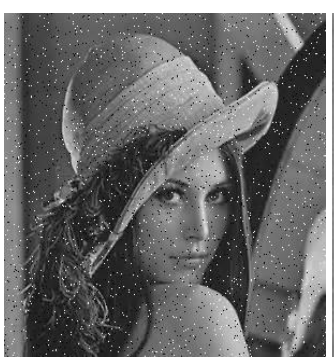

(c)

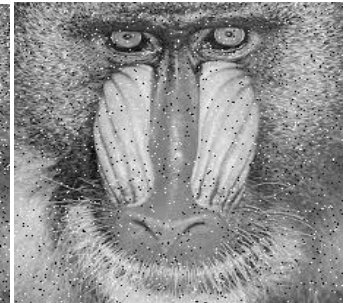

(b)

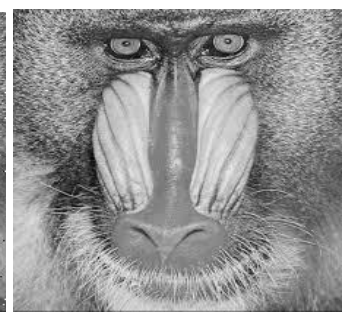

(d)

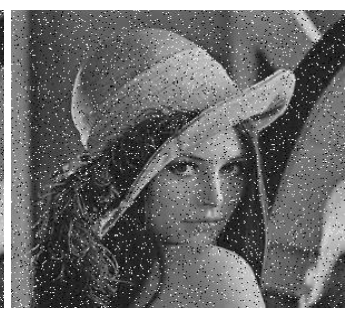

(b)

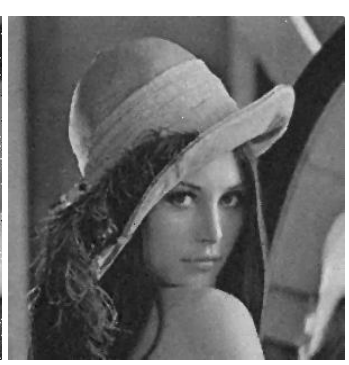

(d)
Fig 4: (a) Corrupted Image (noise is 40\%) (b)MF (c) RMF (d) Proposed filter

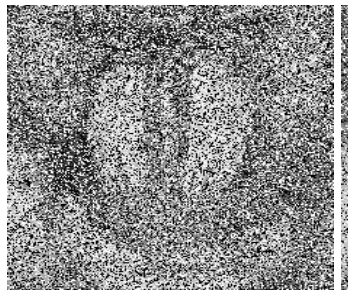

(a)

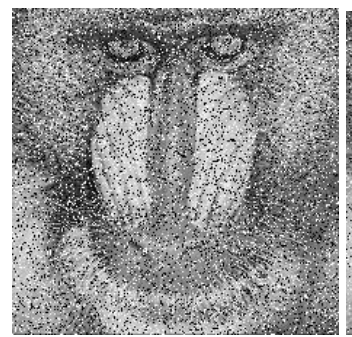

(c)

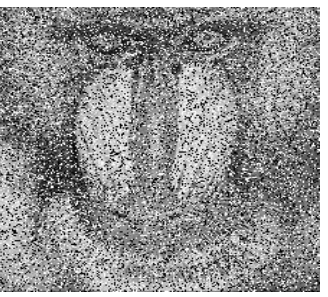

(b)

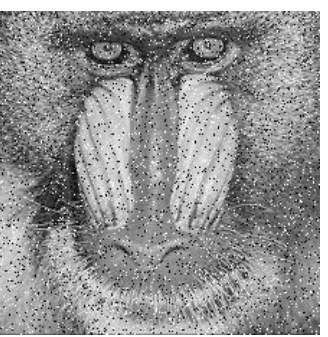

(d)

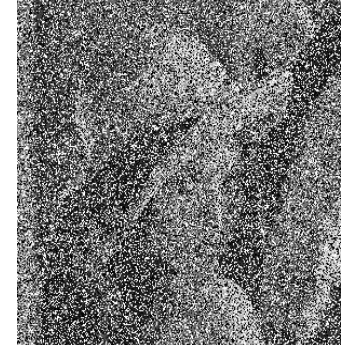

(a)

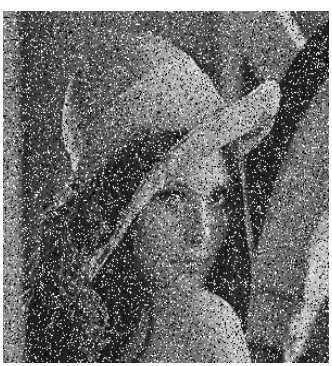

(c)

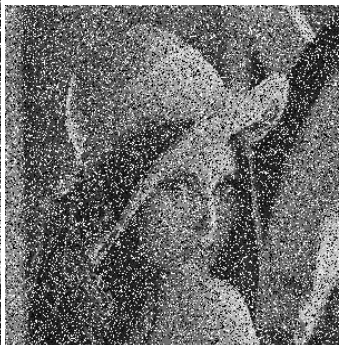

(b)

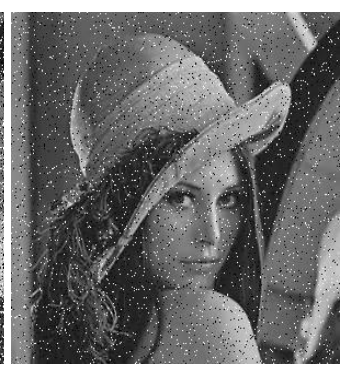

(d)
Fig 5: (a) Corrupted Image (noise is 60\%) (b)MF (c) RMF (d) Proposed filter

\section{REFRENCES}

[1] Mr. Rohit Verma and Dr. Jahid Ali, "A Comparative Study of Various Types of Image Noise and Efficient Noise Removal Techniques", Volume 3, IJARCSSE, ISSN: 2277 128X.

[2] S.Jayaraman, S.Esakkirajan and T. Verakumar, "Digital Image Processing", Tata McGraw Hill, 2009.

[3] R.C. Gonzalez and R.E. Woods, "Digital Image Processing", Third Edition, Practice Hall, 2008.

[4] G. Qiu, "An Improved Recursive Median Filtering Scheme for Image Processing", IEEE Transactions on Image Processing vol.5,no. 4,April 1996.

[5] J. Ishihara, M. Meguro and N. Hamada, "Adaptive weighted median filter utilizing impulsive noise detection," in Applications of Digital Image Processing '99, Proc SPIE 3808, 406-414 (1999).

[6] R.K. Brown Rigg, "The weighted median filter", Commun. Assoc. Comput., pp. 807-818, Mar. 1984.

[7] Vicky Ambale, Minal Ghute, Kanchan, Kambe and Shilpa Ktre , "Adaptive Median filter for image enhancement", International Journal of Engineering Science and Innovative Technology(IJESIT), vol 2, Issue- 1, January 2013.

[8] K. Arakawa, "Median filters based on fuzzy rules and its application to image restoration," Fuzzy Sets Syst., vol. 77, pp. 3-13, 1996.

[9] J. King, "A Novel Recursive Algorithm For DetailPreserving Impulse Noise Removal", Saud University, Vol. 22, Comp. \& Info. Sci., pp. 37-44, Riyadh (1431H./2010). 